\title{
A Mini-Review on the Rehabilitation of Anterior Cruciate Ligament Injury
}

\author{
Caner Kararti and Öznur Büyükturan* \\ School of Physical Therapy and Rehabilitation, Ahi Evran University, Turkey
}

*Corresponding author: Öznur Büyükturan, PT, PhD, Ahi Evran University, School of Physical Therapy and Rehabilitation, Kırşehir, Turkey

Submission: 㘹 June 20, 2018; Published: 㘹 August 17, 2018

\section{Introduction}

The anterior cruciate ligament (ACL) is attached medially to the anterior inter-condylararea of the tibia partly blending with the anterior of the lateral meniscus; it ascends posterolaterally, twisting on itself and fanning out to attach to the posteromedial aspect of the lateral femoral condyle [1]. ACL is the main static stabilizer against anterior translation of the tibia on the femur. Because of oblique anatomy of ACL, it is efficient for limiting excessive anterior tibial translation as well as axial tibial and valgus with knee rotations [2]. Besides its mechanical function related to knee stabilization, there is also neuromuscular function of the ACL due to mechanoreseptors [3]. If there is an ACL deficiency, deafferentation zones can be seen. These zones alter spinal and supra-spinal motor control [4]. The changes in motor control strategy can reveal changes in proprioception, postural control, muscle strength, movement and recruitment patterns [3]. Therefore, an ACL injury is not only a musculoskeletal injury, both also neuro physiological dysfunction.

ACL injuries are reported to be the most common knee ligament injury. It has been estimated that injury rate of 1 per 3,000 in general population [5]. A rehabilitation programme, which promoting the muscular strength and re-establishing the knee joint functional stability, is an essential and integral part of treatment after ACL injury [6]. If exercises are not compatible with normal arthro kinematics of knee, a normal stresses, such as overloading, occur on the tibio femoral joint articulating surfaces and other joint structures [6]. Intrinsic and extrinsic factors related to ACL determine both rehabilitation protocol and return to sports and recruiment activities [7]. Intrinsic factors are genetics /biological characteristics, type of lesion, anatomical features, and compliance with the rehabilitation protocol, motivation, and psychological attitude. As for extrinsic factors, they are type of graft, surgical technique, rehabilitation phases, and biological support [7]. Because of existence of a numerous of factors, rehabilitation protocol should be patient-tailored.

Some factors and functional capacity of the patient in the preoperative period affect the success of the ACL rehabilitation. Preoperative extension deficit, preoperatived eficit in quadriceps strength of $>20 \%$, lack of preoperative rehabilitation are important factors to predict the outcome of treatment [8,9]. Functional recovery of the patient may be worse if mentioned predictive factors are present. Therefore, treatment programme modifications may be needed to achieve the best functional level. Cornerstone of rehabilitation is time of programme and whether the program is supervised rehabilitation or home-based rehabilitation. A minimally supervised rehabilitation programme may result in successful rehabilitation in specific groups of patients that are highly motivated and live far from a physical therapist [10]. Durationof the treatment in either program can be selected to be 19 or 32 weeks. When comparing a 19 -week with a 32 -week rehabilitation programme, there are no differences in terms of laxity, range of motion, self-reported knee function, single-leg hop test for distance or isokinetic concentric quadriceps and harm string muscle strength [3]. The rehabilitation program for ACL should include the following headings: open kinetic chain (OKC) and closed kinetic chain(CKC) exercises, strength training and neuromuscular training, electrostimulation and electromyographic feedback, and cryotherapy.

Andersson et al. [11] Have determined that CKC quadriceps exercises are effective on less pain, less risk of increased laxity and better self-reported knee function compared to OKC quadriceps exercises. Although it has been known that CKC is more beneficial, OKC can also be used. Fukuda et al. [12] described that OKC quadriceps exercises can be started from week 4 after ACL reconstruction with harm string auto greft, but in a limited ROM between $45^{\circ}$ and $90^{\circ}$. An early start to OKC exercises result in more laxity after a follow-up period of 7 months [13-15]. Therefore, it is important for the clinician to train ACL with CKC exercises in early phases of rehabilitation. Although isometric quadriceps exercises are safe from the first post operative week, starting eccentric quadriceps training (in CKC) from 3 weeks after ACL reconstruction is safe and contributes to a bigger improvement in quadriceps strength than concentric training [3]. Neuromuscular training and electro-stimulation/electromyographic feedback in combination with conventional rehabilitation should be added to 
strength training in early phases to optimise self-reported outcome measurements. Regardless of early or late phase of rehabilitation, an important problem that clinicians should eliminate in individuals with ACL lesion is the pain and edema. Cryotherapy is effective in decreasing pain and edema immediately after application upto 1 week ACL post surgery in addition to electirical stimulation applications based on "Gate Control” theory [16].

Return to sports and recruitment activities after ACL lesion is a critical situation which should be correctly timed against possible risk of re-injury. It has been reported that rate of ACL reinjury is $23 \%$ in young individuals [17]. First criteria of return to sports, it has been considered that 6 months as a cut-off value for allowing sport resumption, without major differences emerging between grafts [7]. As regards muscle strength, the cut-off value of $>90 \%$ isokinetic strength compared to the contra lateral side is important criterion most used, followed by lower values of the same parameter $(>85 \%,>80 \%)$ or different parameters, such as a quadriceps index $>90 \%$ and weighted leg extension $>90 \%$ [7]. Selfreport knee scoring (Modified Noyes system $\geq 90$, International Knee Documentation Committee subjective knee form $>70$ ) is frequently used for returning to sports $[18,19]$. In addition to these clinical criteria's, if patient is an athlete, the sport branch of the athlete should also be considered for their turn to the sports [20].

\section{Conclusion}

The decision to allow a patient to return to sport and recruitment activities is a challenge for the clinician. Because there are criteria's both clinic-based and performance-based. It is not possible to form standardised criteria for each patient indiscriminately. It would be preferable to apply patient-tailored rehabilitation protocols and return-to-sport and recruitment activities criteria, based on individual characteristics.

\section{References}

1. Markatos K, Kaseta MK, Lallos SN, Korres DS, Efstathopoulos N (2013) The anatomy of the ACL and its importance in ACL reconstruction. Eur J Orthop Surg Traumatol 23(7): 747-752.

2. Woo SL, Wu C, Dede O, Vercillo F, Noorani S (2006) Biomechanics and anterior cruciate ligament reconstruction. J Orthop Surg Res 1: 2.

3. vanMelick N, vanCingel RE, Brooijmans F, Neeter C, vanTienen $\mathrm{T}$, et al. (2016) Evidence-based clinical practice update: practice guidelines for anterior cruciate ligament rehabilitation based on a systematic review and multidisciplinary consensus. Br J Sports Med 50(24): 1506-1515.

4. Decker LM, Moraiti C, Stergiou N, Georgoulis AD (2011) New insights into anterior cruciate ligament deficiency and reconstruction through the assessment of knee kinematic variability in terms of nonlinear dynamics. Knee Surg Sports Traumatol Arthrosc 19(10): 1620-1633.

5. Sadegh N, Fateme E, Ali S, Reza S, Mohammad A, et al. (2013) Rehabilitation after ACL injury: a fluoroscopic study on the effects of type of exercise on the knees agittal plane arthro kinematics. Bio Med Research International Article ID 248525.
6. Irrgang JJ, Fitzgerald GK (2000) Rehabilitation of the multiple ligament-injured knee. Clin Sports Med 19(3): 543-571.

7. Zaffagnini S, Grassi A, Serra M, Marcacci M (2015) Return to sport after ACL reconstruction: how, when and why? A narrative review of current evidence. Joints 3(1): 25-30.

8. de Valk EJ, Moen MH, Winters M, Bakker EW, Tamminga R (2013) Preoperative patient and injury factors of successful rehabilitation after anterior cruciate ligament reconstruction with single-bundle techniques. Arthroscopy 29: 1879-1895.

9. Eitzen I, Holm I, Risberg MA (2009) Preoperative quadriceps strength is a significant predictor of knee function two years after anterior cruciate ligament reconstruction. Br J Sports Med 43(5): 371-376.

10. Wright RW1, Preston E, Fleming BC, Amendola A, Andrish JT, et al (2008) A systematic review of anterior cruciate ligament reconstruction rehabilitation. Part I: continuous passive motion, early weight bearing, postoperative bracing, and home-based rehabilitation. J Knee Surg 21(3): 217-224.

11. Andersson D, Samuelsson K, Karlsson J (2009) Treatment of anterior cruciate ligament injuries with special reference to surgical technique and rehabilitation: an assessment of randomized controlled trials. Arthroscopy 25(6): 653-685.

12. Fukuda TY, Fingerhut D, Moreira VC, Camarini PM, Scodeller NF, et al. (2013) Open kinetic chain exercises in a restricted range of motion after anterior cruciate ligament reconstruction. A randomized controlled trial. Am J Sports Med 41(4): 788-794.

13. Heijne A, Werner S (2007) Early versus late start of open kinetic chain quadriceps exercises after ACL reconstruction with patellar tendon or hamstring grafts: a prospective randomized outcome study. Knee Surg Sports Traumatol Arthrosc 15(4): 402-414.

14. Kruse LM, Gray B, Wright RW (2012) Rehabilitation after anterior cruciate ligament reconstruction. A systematic review. J Bone Joint Surg Am 94(19): 1737-1748

15. Imoto AM, Peccin S, Almeida GJ, Saconato H, Atallah ÁN (2011) Effectiveness of electrical stimulation on rehabilitation after ligament and meniscal injuries: a systematic review. Sao Paulo Med J 129(6): 414-423.

16. Raynor MC1, Pietrobon R, Guller U, Higgins LD (2005) Cryotherapy after ACL reconstruction: a meta-analysis. J Knee Surg. 18(2): 123-129.

17. Wiggins AJ, Grandhi RK, Schneider DK, Stanfield D, Webster KE, et al. (2016) Risk of secondary injury in younger athletes after anterior cruciate ligament reconstruction: a systematic review and meta-analysis. Am J Sports Med 44(7): 1861-1876.

18. Wilk KE1, Arrigo C, Andrews JR, Clancy WG (1999) Rehabilitation after anterior cruciate ligament reconstruction in the female athlete. J Athl Train 34(2): 177-193.

19. Myer GD, Paterno MV, Ford KR, Quatman CE, Hewett TE (2006) Rehabilitation after anterior cruciate ligament reconstruction: criteria-based progression through the return-to-sport phase. J Orthop Sports Phys Ther 36(6): 385-402.

20. Rambaud AJM, Ardern CL, Thoreux P, Regnaux JP, Edouard P (2018) Criteria for return for running after anterior cruciate ligamentre construction: a scoping review. Br J Sports Med pii: bjsports-2017-098602. 
Creative Commons Attribution 4.0 International License

For possible submissions Click Here
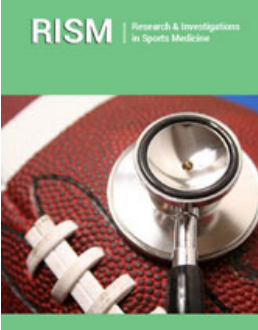

Research \& Investigations in Sports Medicine

\section{Benefits of Publishing with us}

- High-level peer review and editorial services

- Freely accessible online immediately upon publication

- Authors retain the copyright to their work

- Licensing it under a Creative Commons license

- Visibility through different online platforms 